Linguistic Description Moderates the Evaluations of Counterstereotypical People

Mark Rubin and Stefania Paolini

The University of Newcastle, Australia

Richard J. Crisp

The University of Kent, UK

The correct reference for this article is as follows:

Rubin, M., Paolini, S., \& Crisp, R. J. (2013). Linguistic description moderates the evaluations of counterstereotypical people. Social Psychology, 44, 289-298. doi: $\underline{10.1027 / 1864-9335 / a 000114}$

This self-archived version is provided for non-commercial, scholarly purposes only.

This research was supported by the Australian Research Council's Discovery Project funding scheme (DP0556908). We are grateful to the Study Response Project team for their assistance with data collection.

Correspondence concerning this article should be addressed to Mark Rubin at the School of Psychology, The University of Newcastle, Callaghan, NSW 2308, Australia. Tel: +61 (0)2 4921 6706. Fax: +61 (0)2 4921 6980. E-mail: Mark.Rubin@ @ewcastle.edu.au 
Abstract

The present research investigated linguistic description as a moderator of biased evaluations of counterstereotypical individuals. Members of an online participant pool $(N=237)$ indicated their liking for stereotypical and counterstereotypical individuals who were described using adjectives or behaviors. There was a significant interaction between target typicality and linguistic description: People liked counterstereotypical individuals more than stereotypical individuals when target individuals were described using adjectives. In contrast, they showed no bias or a negative bias against counterstereotypical individuals who were described using behaviors. This interaction effect generalized across gender targets (men/women) and sexuality targets (gay/straight), and it was partially mediated by subjective processing fluency. Implications for the backlash effect and prejudice reduction are discussed.

KEYWORDS: stereotypical, counterstereotypical, processing fluency, backlash effect, category-expectancy violation, shifting standards 


\section{Linguistic Description Moderates the Evaluations of Counterstereotypical People}

We use stereotypes to facilitate our understanding of people from a large and diverse array of social groups (for a review, see Hilton \& von Hippel, 1996). For example, people might stereotype Asian students as intelligent and blonde women as unintelligent. But how do people feel about individuals who buck the trend and contradict their stereotypes? For example, how do people feel about unintelligent Asians and clever blondes?

There is some evidence that people tend to react quite negatively towards counterstereotypical individuals, showing a so-called backlash effect against them (Cohen, Hall, \& Tuttle, 2009; Gill, 2004; Okimoto \& Brescoll, 2010; Rudman, 1998; Rudman \& Glick, 1999, 2001; Rudman \& Fairchild, 2004; see also Schimel et al., 1999). The typical explanation for this prejudice refers to people's need to protect and maintain their stereotypes (e.g., Moreno \& Bodenhausen, 1999; Rudman \& Fairchild, 2004; Schimel et al., 1999): People tend to be prejudiced against counterstereotypical individuals because counterstereotypical individuals disconfirm stereotypes and threaten people's need to maintain stable and coherent stereotype systems.

However, previous research has also identified several conditions under which counterstereotypical individuals may be evaluated positively. In the present article, we briefly discuss this evidence and then consider linguistic description as a potential new moderator of evaluations counterstereotypical individuals.

\section{Established Moderators of Evaluations of Counterstereotypical Individuals}

Research on category-based expectancy violation (e.g., Bettencourt, Dill, Greathouse, Charlton, \& Mulholland, 1997) indicates that individuals from low status groups who have positive attributes (e.g., a black college applicant with high grades) are evaluated more positively than individuals from high status groups who have positive attributes (e.g., a white college applicant with high grades) despite both individuals having the same attributes (high grades). However, this effect also works the other way around. Hence, individuals from high status groups who have negative attributes (e.g., a white applicant with low grades) are evaluated more negatively than individuals from low status groups who have negative attributes (e.g., a black applicant with low grades). In general then, counterstereotypical individuals may be evaluated either more positively or more negatively than stereotypical individuals depending on an interaction between their valence and their group's valence.

Similar to the category-based expectancy violation effect, the shifting standards model (e.g., Biernat, Manis, \& Nelson, 1991) holds that people adjust their standards when making subjective ratings of counterstereotypical individuals. Again, this effect can sometimes lead to relatively positive evaluations of counterstereotypical individuals. So, for example, people are likely to rate a man as more "caring and nurturing" than a woman after observing both individuals perform the same caring and nurturing behavior (e.g., looking after a child). This effect occurs because people adopt different, stereotype-based standards for what constitutes caring and nurturing behavior when judging men and women. Again, this effect can work the other way around. Hence, people are likely to rate a man as more "nagging" than a woman when both individuals perform the exactly the same nagging behavior. Hence, similar to the category-based expectancy violation model, the shifting standards model predicts that counterstereotypical individuals may be evaluated either more positively or more negatively than stereotypical individuals depending on an interaction between the individuals' characteristics and their group's stereotype.

Finally, Gutierrez and Unzueta (2010) have recently demonstrated that interethnic ideologies can moderate the evaluation of counterstereotypical individuals. In this research, participants read a story that promoted the ideologies of either colorblindness or multiculturalism. Participants then evaluated the Facebook profiles of counterstereotypical 
and stereotypical Black and Latino individuals. Participants in the colorblindness condition liked counterstereotypical individuals more than stereotypical individuals. The authors speculated that this is because counterstereotypical individuals confirm the colorblindness assumption that group boundaries are relatively permeable. Participants in the multiculturalism condition liked stereotypical individuals more than counterstereotypical individuals. The researchers speculated that this was because counterstereotypical individuals are not good representatives of their group and so do not offer the opportunity to learn about the out-group - a key aspect of multiculturalism. Overall, this research provides evidence that evaluations of counterstereotypical individuals can depend on salient social ideologies.

\section{Uniqueness - A Further Reason to Like Counterstereotypical Individuals?}

In the present research, we consider a novel reason why people may like counterstereotypical individuals and a novel moderator of their evaluations. Specifically, we consider counterstereotypical individuals' uniqueness as a potentially positive aspect of their counterstereotypicality and linguistic description as a potential moderator of the influence of this aspect on evaluations.

Counterstereotypical people are, by definition, relatively unique individuals, and Westerners like unique stimuli more than typical stimuli. This preference for uniqueness has been demonstrated most clearly by Kim and Markus (1999). In two studies, these researchers asked participants to indicate their preference for nine geometric shapes. In each set, one or more shapes varied in terms of its shape, direction, or position relative to the others (e.g., two triangles and seven circles). The researchers found that European Americans preferred atypical shapes more than typical shapes. In a third study, research assistants offered participants one of five pens, one or two of which were of a different color to the others. Again, American participants tended to choose a uniquely-colored pen. Kim and Markus concluded that American culture includes "the glorification of individual freedom to reject norms and an aversion to conformity" (p. 795).

The cultural glorification of individuality has been found to affect not only evaluations of shapes and pens, but also social motivation and influence. Westerners have a general desire to be distinctive and personally unique (Brewer, 1991; Lynn \& Snyder, 2002; Snyder \& Fromkin, 1977, 1980), and this need for uniqueness leads them to perceive themselves as being less conformist than others (Pronin, Berger, \& Molouki, 2007) and to resist conformity (Imhoff \& Erb, 2009).

Based on this evidence, Westerners have the potential to like counterstereotypical individuals more than stereotypical individuals because counterstereotypical individuals symbolize the positive cultural values of uniqueness and nonconformity. In the present research, we investigated linguistic description as a potential moderator of the extent to which the uniqueness of counterstereotypical individuals becomes apparent.

\section{The Moderating Role of Linguistic Description}

We hypothesized that linguistic description operates as a moderator of evaluations of counterstereotypical individuals. We predicted that counterstereotypical individuals would be evaluated relatively positively when they are described using adjectives because this linguistic description promotes heuristic processing which is influenced by the idea that uniqueness is good. We also predicted that counterstereotypical individuals would be evaluated relatively negatively when they are described using behaviors because this linguistic description promotes deeper, more systematic processing which highlights the negative aspects of stereotype disconfirmation. We based these predictions on previous theorizing and research by ter Doest, Semin, and Sherman (2002). 
Ter Doest et al. (2002) considered the effect of linguistic description on the inconsistency resolution process that occurs when people are exposed to a counterstereotypical individual (e.g., Coull, Yzerbyt, Castano, Paladino, \& Leemans, 2001; Hutter \& Crisp, 2005; Kunda, Miller, \& Claire, 1990). When people encounter an individual whose characteristics are inconsistent with stereotypical expectations, they search for similar inconsistent information in memory and, if they do not find this information, they engage in casual reasoning to explain the inconsistency (Kunda et al., 1990). Importantly, this inconsistency resolution process is thought to require considerable cognitive effort (GarciaMarques \& Mackie, 1999, p. 985; Hutter \& Crisp, 2005, 2006).

In considering how linguistic description might affect the inconsistency resolution process, ter Doest et al. (2002, p. 200) assumed that abstract language, such as adjectival descriptions, elicits a superficial level of processing that is influenced by heuristics. In contrast, concrete language, such as behavioral descriptions, elicits a deeper and more systematic level of processing that is less likely to be influenced by heuristics. Based on these assumptions, ter Doest et al. proposed that counterstereotypical individuals are most likely to trigger an inconsistency resolution process when they are described using behaviors rather than adjectives because behaviors are more likely to elicit the cognitive engagement that is necessary for this process to occur.

Consistent with these predictions, ter Doest et al. (2002, Study 1) found that linguistic description (adjectival/behavioral) moderated the effect of target typicality

(stereotypical/counterstereotypical) on processing style: Participants paid significantly more attention to counterstereotypical individuals than to stereotypical individuals when targets were described using behaviors. However, this difference became nonsignificant when targets were described using adjectives. Importantly, however, ter Doest et al. did not investigate whether either linguistic description or processing style affected participants' evaluations of counterstereotypical individuals. The present research addresses this important gap in the literature.

\section{Summary of Predictions}

Following ter Doest et al. (2002), we assumed that adjectival descriptions would elicit a heuristic processing style and that, under this processing style, evaluations of stereotypical and counterstereotypical individuals would be influenced by the heuristic that "uniqueness is good" (Kim \& Markus, 1999). Given that counterstereotypical individuals are more unique than stereotypical individuals, we predicted that adjectival descriptions should elicit a bias in favor of counterstereotypical individuals.

In contrast, we assumed that behavioral descriptions would elicit a more systematic processing style and that, under this processing style, evaluations of stereotypical and counterstereotypical individuals would be influenced by an inconsistency resolution process that highlights the negative aspects of inconsistency for stereotype coherence. Given that counterstereotypical individuals are more inconsistent than stereotypical individuals, we predicted that behavioral descriptions should elicit a bias against counterstereotypical individuals.

In order to test the influence of process style, we included a measure of subjective processing fluency in our research (Rubin, Paolini, \& Crisp, 2010). Processing fluency can be considered as a characteristic of stimuli, and it is often measured via response latencies (e.g., Winkielman, Halberstadt, Fazendeiro, \& Catty, 2006). Our measure of subjective processing fluency related to the ease or difficulty that people felt when attempting to imagine target individuals. Given that systematic processing is more subjectively difficult than heuristic processing, we expected this measure to be a valid measure of processing style. Following ter Doest et al. (2002), we predicted that participants would find it relatively more 
difficult to think about counterstereotypical individuals when they were described using behaviors rather than adjectives. We also predicted that processing fluency would mediate the moderating effects of linguistic description on evaluations of stereotypical and counterstereotypical individuals.

\section{Overview of the Present Research}

In the present research, participants indicated their liking for stereotypical and counterstereotypical individuals who were described using adjectives or behaviors. In order to test the generalizability of our results, we represented target individuals using two different social groups: gender (male/female) and sexuality (gay/straight). We chose these specific target groups because they represent important bases for social categorization, and they are each associated with clear stereotypes.

We were conscious that our participants might not express negative evaluations of target individuals because of concerns about social desirability, political correctness, and maintaining a nonprejudiced self-image (e.g., Crandall \& Eshleman, 2003; Holmes, 2009). We used two methods to address this potential problem. First, we assessed ratings of stereotypical and counterstereotypical targets using a between-subjects design. Hence, participants responded to either stereotypical individuals or counterstereotypical individuals. This approach reduced the contrast between stereotypical and counterstereotypical targets for each participant and therefore reduced the potential accessibility of our hypotheses of evaluative biases in relation to these types of target individuals. Second, we used a cover story to divert participants' attention from the evaluative aspect of our research. Specifically, we informed participants that our research was investigating "how familiar different kinds of people appear". In order to corroborate this cover story, we titled our research "Familiar People?", and we included a measure of familiarity in our research. We debriefed participants about this minor deception and informed them about the true purpose of the research after they had completed the research.

\section{Participants and Research Design}

\section{Method}

We recruited participants from a US-based online participant pool called the Study Response Project. Participants were entered into a prize draw to receive US\$100 gift certificates from an online store.

We collected data from 274 participants. Data from 37 participants were excluded because the participants (a) did not finish the questionnaire and indicate their informed consent $(n=27)$, (b) finished the questionnaire but declined their informed consent $(n=2)$, or (c) finished the questionnaire and indicated their consent but had large amounts of missing data $(>15 \%)$ in their questionnaire $(n=8)$.

The final sample consisted of 237 people (95 men and 141 women; one participant declined to indicate their gender) who had a mean age of 38.83 years ( $S D=11.40$ years). The majority of participants $(80.7 \%)$ were employed. The research design consisted of a 2 (target typicality: stereotypical/counterstereotypical) x 2 (linguistic description: adjectival/behavioral) x 2 (group type: gender/sexuality) mixed-model design with repeated measures on the last two factors. One hundred and eighteen participants responded to stereotypical targets, and 119 participants responded to counterstereotypical targets.

\section{Procedure and Measures}

The administrators of the Study Response Project contacted prospective participants by e-mail and asked them to click on an internet link if they wished to participate. The link took participants to our questionnaire, which was hosted by a survey design company called 
PsychData. PsychData software randomly assigned participants to complete a questionnaire that referred to either eight stereotypical individuals or eight counterstereotypical individuals. ${ }^{1}$

In both conditions, four of the target individuals were based on gender ("a man" and "a woman"), and four were based on sexuality ("a straight man" and "a gay man"). Previous research has confirmed the typicality of the adjectives that we used to describe gender targets ("aggressive" and "sensitive"; Maass, Cadinu, Boni, \& Borini, 2005; Rubin, Hewstone, \& Voci, 2001, Study 2) and sexuality targets ("insensitive" and "creative"; Simon, GlässnerBayerl, \& Stratenwerth, 1991). We generated stereotypical and counterstereotypical behaviors ourselves for gender targets ("knitting" and "fishing") and for sexuality targets ("in a sexual relationship with a man" and "in a sexual relationship with a woman"). Hence, example descriptions of target individuals included "a man who is fishing" (a behavioral description of a stereotypical gender target) and "an insensitive gay man" (an adjectival description of a counterstereotypical sexuality target).

For each of the eight target individuals, participants responded to five items on 7point Likert-type rating scales. Consistent with our cover story, the first item asked participants to indicate how familiar the target individual was (very unfamiliar, very familiar). The next two items asked participants to indicate how stereotypical (very counterstereotypical, very stereotypical) and conventional (very unconventional, very conventional) the target individual was. These two items were included in order to confirm the perceived typicality of the target individuals. The next item asked participants to indicate how easy it was to imagine the target individual (very difficult, very easy). This item was included as a measure of subjective processing fluency, and it represented our potential mediator variable. The last item asked participants to rate how likable the person was (very dislikable, very likable). This item represented our dependent variable. ${ }^{2}$

\section{Eliminating Potentially Confounding Effects}

\section{Results}

Participants' evaluations of target individuals had the potential to be influenced by four effects that could confound our interpretation of the results. First, evaluations might be influenced by the valence of the particular descriptions that we used. For example, participants might like a sensitive man more than an aggressive man because sensitivity is more socially desirable than aggressiveness.

Second, evaluations might be influenced by the in-group/out-group status of the targets' social groups (for a review, see Hewstone, Rubin, \& Willis, 2002). For example, male participants might like a sensitive man more than a sensitive woman because the man represents an in-group member and the woman represents an out-group member. As another example, heterosexual participants might like a creative straight man more than a creative gay man because the straight man represents an in-group member and the gay man represents an out-group member.

Third, evaluations might be influenced by the social status of the target individuals' social groups (for a review, see Mullen, Brown, \& Smith, 1992). For example, participants might like a sensitive man more than a sensitive woman because they perceive men to have a higher social status than women (e.g., Eagly \& Wood, 1982).

Fourth, target individuals' positive or negative valence might interact with their social group's status to influence evaluations via a category-based expectancy violation effect (e.g., Bettencourt et al., 1997). For example, participants might like a sensitive man more than an aggressive man because sensitivity is a positive trait that violates participants' negative expectations about men based on their stereotype of being aggressive. 
To ensure that our results were not influenced by these potentially confounding effects, we did not analyze participants' ratings of individual targets. So, for example, we did not test whether people rated "an aggressive man" (stereotypical) more or less positively than "a sensitive man" (counterstereotypical) because this type of comparison is very likely to be influenced by the valence of the specific traits that we used (i.e., "aggressive" is more negative than "sensitive"). Instead, we used an approach based on Schimel et al. (1999, Study 4) in which we computed the average of each participant's ratings of target individuals within each typicality type (stereotypical/counterstereotypical). ${ }^{3}$ So, for example, we computed the average of each participant's ratings of "an aggressive man" and "a sensitive woman" in order to obtain mean ratings for stereotypical gender targets who are described using traits. Likewise, we computed the average of each participant's ratings of "a sensitive man" and "an aggressive woman" in order to obtain mean ratings for counterstereotypical gender targets who are described using traits. We then compared these two mean ratings in order to identify evaluative biases in relation to stereotypical and counterstereotypical individuals independent of the influence of the abovementioned potentially confounding effects. So, for example, if a participant's average evaluation of "an aggressive man" and "a sensitive woman" (both stereotypical) is more positive than their average evaluation of "a sensitive man" and "an aggressive woman" (both counterstereotypical), then this evaluative bias cannot be due to differences in the valence of the specific traits that were used ("sensitive" and "aggressive") because the average valence of these two traits in the stereotypical description - (("aggressive" + "sensitive")/2) - is exactly identical to the average valence of these two traits in the counterstereotypical description - (("aggressive" + "sensitive")/2). The same principle applies equally well to potentially confounding effects that are based on in-group favoritism, group status, category-based expectancy violation, and any other variables that are associated with the specific traits and individuals that we used to describe stereotypical and counterstereotypical individuals. ${ }^{4}$

\section{Check on Target Typicality}

The ratings of stereotypicality and conventionality were internally consistent ( $\alpha$ s ranged from .66 to .83) for seven of the eight description types based on target typicality (stereotypical/counterstereotypical), linguistic description (adjectival/behavioral), and group type (gender/sexuality). The alpha for counterstereotypical sexuality-based targets who were described using adjectives was lower than desirable $(\alpha=.57)$. Nonetheless, we computed mean stereotypicality and conventionality ratings in order to create an overall typicality index for each of the four types of target individuals.

A series of independent samples $t$ tests confirmed that stereotypical individuals were rated as being significantly more typical than counterstereotypical individuals for all four types of target individuals, $p s<.001$. Hence, we confirmed the validity of the adjectives and behaviors that we chose to describe stereotypical and counterstereotypical individuals. ${ }^{5}$

\section{Investigating the Evaluation of Target Individuals}

Using liking as a dependent variable, we performed a 2 (target typicality: stereotypical/counterstereotypical) x 2 (linguistic description: adjectival/behavioral) x 2 (group type: gender/sexuality) mixed-model ANOVA with repeated measures on the last two factors. ${ }^{6}$ This ANOVA revealed significant main effects of linguistic description and group type as well as significant two-way interactions between target typicality and linguistic description, target typicality and group type, and linguistic description and group type (all $p \mathrm{~s}$ $<.001)$. These effects were qualified by a significant three-way interaction between target typicality, linguistic description, and group type, $F(1,235)=19.16, p<.001, \eta_{\mathrm{p}}{ }^{2}=.08$. 
To decompose this three-way interaction, we examined the simple two-way interaction effect between target typicality and linguistic description at each level of group type. This simple two-way interaction was significant for both gender targets, $F(1,235)=$ $9.59, p=.002, \eta_{\mathrm{p}}{ }^{2}=.04$, and sexuality targets, $F(1,235)=77.87, p<.001, \eta_{\mathrm{p}}{ }^{2}=.25$, although it was a substantially larger effect in the latter case. We decomposed each simple two-way interaction by conducting simple comparisons between stereotypical and counterstereotypical targets at each level of linguistic description.

When gender targets were described using adjectives, participants liked counterstereotypical targets $(M=4.73)$ significantly more than stereotypical targets $(M=$ 4.17), $F(1,235)=14.94, p<.001, \eta_{\mathrm{p}}{ }^{2}=.06$. In contrast, when gender targets were described using behaviors, there was no significant difference in evaluations of stereotypical targets $(M$ $=5.40)$ and counterstereotypical targets $(M=5.41), F(1,235)=.01, p=.922, \eta_{\mathrm{p}}{ }^{2}<.01$.

When sexuality targets were described using adjectives, participants liked counterstereotypical targets $(M=4.51)$ significantly more than stereotypical targets $(M=$ 4.14), $F(1,235)=6.31, p=.013, \eta_{\mathrm{p}}^{2}=.03$. In contrast, when sexuality targets were described using behaviors, participants liked stereotypical targets $(M=5.11)$ significantly more than counterstereotypical targets $(M=3.87), F(1,235)=48.38, p<.001, \eta_{\mathrm{p}}{ }^{2}=.17$.

\section{Investigating the Processing Fluency of Target Individuals}

Following ter Doest et al. (2002), we predicted that participants would find it more difficult to think about counterstereotypical individuals than stereotypical individuals when targets were described using behaviors rather than adjectives. To test this prediction, we used processing fluency as a dependent variable and performed a 2 (target typicality: stereotypical/counterstereotypical) x 2 (linguistic description: adjectival/behavioral) x 2 (group type: gender/sexuality) mixed-model ANOVA with repeated measures on the last two factors.

The ANOVA revealed significant main effects of target typicality, linguistic description, and group type as well as significant two-way interactions between target typicality and linguistic description, target typicality and group type, and linguistic description and group type (all $p s<.001$ ). These effects were qualified by a significant threeway interaction between target typicality, linguistic description, and group type, $F(1,235)=$ $13.15, p<.001, \eta_{\mathrm{p}}^{2}=.05$.

To decompose this three-way interaction, we examined the simple two-way interaction effect between target typicality and linguistic description at each level of group type. This simple two-way interaction was significant for both gender targets, $F(1,235)=$ $22.04, p<.001, \eta_{\mathrm{p}}{ }^{2}=.09$, and sexuality targets, $F(1,235)=69.21, p<.001, \eta_{\mathrm{p}}{ }^{2}=.23$, although it was a substantially larger effect in the latter case. Again, we decomposed each simple two-way interaction by conducting simple comparisons between stereotypical and counterstereotypical targets at each level of linguistic description.

Consistent with ter Doest et al.'s (2002) results, when gender targets were described using adjectives, there was no significant difference in the difficulty that participants had in thinking about stereotypical targets $(M=5.91)$ and counterstereotypical targets $(M=5.87)$, $F(1,235)=.12, p=.73, \eta_{\mathrm{p}}^{2}<.01$. In contrast, when gender targets were described using behaviors, participants found it significantly more difficult to think about counterstereotypical targets $(M=5.02)$ than stereotypical targets $(M=5.80), F(1,235)=$ $22.88, p<.001, \eta_{\mathrm{p}}^{2}<.09$.

For sexuality targets, simple comparisons revealed that participants found it significantly more difficult to consider counterstereotypical targets than stereotypical targets when targets were described using both adjectives $\left(M_{\text {Stereotypical }}=5.74, M_{\text {Counterstereotypical }}=\right.$ $5.24), F(1,235)=8.26, p=.004, \eta_{\mathrm{p}}{ }^{2}=.03$, and behaviors $\left(M_{\text {Stereotypical }}=5.61\right.$, 
$\left.M_{\text {Counterstereotypical }}=3.58\right), F(1,235)=116.01, p<.001, \eta_{\mathrm{p}}{ }^{2}=.33$. Hence, the main effect of target typicality dominated these simple comparisons. Nonetheless, consistent with ter Doest et al. (2002), the size of this effect was substantially smaller in relation to adjectival descriptions $\left(\eta_{\mathrm{p}}^{2}=.03\right)$ than it was in relation to behavioral descriptions $\left(\eta_{\mathrm{p}}{ }^{2}=.33\right)$.

\section{Investigating the Mediating Effects of Processing Fluency in Evaluations of Target Individuals}

Given the supportive pattern of results from the processing fluency data, we proceeded with a mediated moderation analysis (Baron \& Kenny, 1986; Muller, Judd, \& Yzerbyt, 2005). We have already demonstrated the moderating effect of linguistic description on (a) evaluations and (b) processing fluency of stereotypical and counterstereotypical individuals. In a final analysis, we investigated the moderating effect of linguistic description on evaluations of stereotypical and counterstereotypical targets after controlling for processing fluency.

We conducted our mediated moderation analysis using a mixed-model ANCOVA rather than a multiple regression because one of our independent variables (linguistic description) was a repeated measures variable. ANCOVA is an appropriate statistical method for conducting mediation analyses (Yzerbyt, Muller, \& Judd, 2004), and it has been used to test mediation effects in previous research in this area (ter Doest et al., 2002, p. 207).

Given the two-way interaction between target typicality and linguistic description was qualified by group type, we conducted one ANCOVA for gender targets and one ANCOVA for sexuality targets. These ANCOVAs consisted of a 2 (target typicality: stereotypical/counterstereotypical) x 2 (linguistic description: adjectival/behavioral) mixedmodel design with repeated measures on the last factor, liking as the dependent variable, and the centered summed score of processing fluency on adjectives and behaviors as a covariate. ${ }^{8}$

For gender groups, the ANCOVA showed that the two-way interaction between target typicality and linguistic description that we had obtained in our previous analyses, $F(1,235)$ $=9.59, p=.002, \eta_{\mathrm{p}}{ }^{2}=.04$, remained significant, $F(1,234)=5.49, p=.02, \eta_{\mathrm{p}}{ }^{2}=.02$, but as a marginally weaker effect (Sobel's $Z=1.87, p=.062$ ). In addition, there was a significant main effect of processing fluency, $F(1,234)=60.39, p<.001, \eta_{\mathrm{p}}{ }^{2}=.21$, and a significant two-way interaction between linguistic description and processing fluency, $F(1,234)=15.70$, $p<.001, \eta_{\mathrm{p}}^{2}=.06$.

For sexuality groups, the ANCOVA showed that the previously-obtained two-way interaction between target typicality and linguistic description, $F(1,235)=77.87, p<.001$, $\eta_{\mathrm{p}}{ }^{2}=.25$, remained significant, $F(1,234)=37.54, p<.001, \eta_{\mathrm{p}}{ }^{2}<.14$, but as a significantly weaker effect (Sobel's $Z=5.03, p<.001$ ). In addition, there was a significant main effect of processing fluency, $F(1,234)=99.73, p<.001, \eta_{\mathrm{p}}{ }^{2}=.30$, and a significant two-way interaction between linguistic description and processing fluency, $F(1,234)=37.54, p<$ $.001, \eta_{\mathrm{p}}{ }^{2}=.14$. The pattern of results for both gender and sexuality targets fulfils the criteria for partially mediated moderation effects (Muller et al., 2005, p. 856). ${ }^{8}$

\section{Discussion}

\section{Summary of Key Findings}

The present research identified a previously unreported moderating effect of linguistic description on evaluations of stereotypical and counterstereotypical individuals. This moderating effect generalized across target individuals based on gender groups and sexuality groups, although it was stronger in the latter case. For gender targets, participants evaluated counterstereotypical individuals relatively positively when they were described using adjectives, and this positive bias became nonsignificant when targets were described using behaviors. For sexuality targets, participants evaluated counterstereotypical individuals 
relatively positively when they were described using adjectives, and this bias reversed to become a negative bias against counterstereotypical individuals when targets were described using behaviors.

The difference in the pattern of the interaction effect for gender and sexuality targets may be related to the particular stimulus materials that we used. Although participants always found it more difficult to think about counterstereotypical individuals than stereotypical individuals when targets were described using behaviors, this effect was substantially larger for sexuality targets $\left(\eta_{\mathrm{p}}{ }^{2}=.33\right)$ than for gender targets $\left(\eta_{\mathrm{p}}{ }^{2}=.09\right)$. Hence, sexuality targets may have elicited a more intensive inconsistency resolution process than gender targets. Future research should test the generalizability of our findings to other stimulus materials and target groups in order to investigate this issue.

We should note that the difference in the pattern of the interaction effect for gender and sexuality targets does not invalidate or confound the moderating effect of linguistic description. The key point from our perspective is that the difference in evaluations of stereotypical and counterstereotypical individuals showed a significant and theoreticallyconsistent change as a function of linguistic description for both gender-based targets and sexuality-based targets. In other words, although the moderating effect of linguistic description was larger for sexuality-based targets than for gender-based targets, it was nonetheless significant in both cases. Consequently, our results demonstrate the generality of this effect across different target groups and descriptions.

Importantly, in-group bias, group status, description valence, and category-based expectancy violation effects all had the potential to influence our results. However, we precluded the influence of these potential effects in our analyses by counterbalancing them across the target typicality factor (stereotypical/counterstereotypical). This approach rendered these effects untenable as potential explanations for our results. To be clear, we acknowledge that these effects are likely to be influential in the real world. Our point is only that, due to the counterbalanced approach that we employed, these effects cannot explain the interactions between target typicality and linguistic description that we obtained.

Following ter Doest et al. (2002), we assumed that processing style was partly responsible for the moderating effect of linguistic description on evaluations of stereotypical and counterstereotypical individuals. Consistent with this processing style explanation and ter Doest et al.'s (2002) evidence, participants found it more difficult to imagine counterstereotypical individuals than stereotypical individuals when targets were described using behaviors rather than adjectives. Furthermore, subjective processing fluency partially mediated the two-way interaction between target typicality and linguistic description for both gender targets and sexuality targets.

\section{Implications and Directions for Future Research}

The present research adds to the research in this area in several ways. First, the research extends the work of ter Doest et al. (2002) by demonstrating that adjectival and behavioral descriptions lead to not only different styles of processing counterstereotypical individuals, but also different evaluations of counterstereotypical individuals. Future research may wish to test the generalizability of this moderating effect of linguistic description to different measures of evaluation (e.g., implicit measures; Biernat, Collins, Katzarska-Miller, \& Thompson, 2009).

Our findings also add to previous research that has shown that evaluations of counterstereotypical individuals may be influenced by category-based expectancy violation (e.g., Bettencourt et al., 1997), shifting standards (e.g., Biernat et al., 1991), and intergroup ideologies (Gutierrez \& Unzueta, 2010). This previous research has found that evaluations of counterstereotypical individuals depend on the specific relations that they have with their 
group's stereotypes or broader intergroup ideologies. In contrast, the present research demonstrates that evaluations of counterstereotypical individuals can also depend on their linguistic description and the ease with which they are processed.

Our research findings imply a potential limiting factor for the backlash effect. The typical demonstration of this effect has shown that people rate counterstereotypical female job applicants as less socially skilled and less hirable than stereotypical male applicants (Rudman, 1998; Rudman \& Fairchild, 2004; Rudman \& Glick, 1999, 2001). Importantly, backlash researchers have manipulated target typicality via behavioral descriptions and not via adjectival descriptions. For example, they have manipulated the typicality of male or female confederates' behavior during an interview (Rudman, 1998; Rudman \& Glick, 1999, 2001). Consequently, the backlash effect may be limited to behavioral descriptions and may not generalize to adjectival descriptions (for a similar point, see ter Doest et al., 2002, p. 222). Future research on the backlash effect should investigate this issue further.

Our research findings are consistent with previous models that have considered the influence of heuristic and systematic processing on stereotyping (e.g., Brewer, 1988). As Brewer and Feinstein (1999) explained, stereotypes can be processed either heuristically or systematically and, consistent with our assumptions, "with more effortful processing, data about the individual will be matched against features of the category prototype [and] mismatches will be identified" (p. 258). Hence, although systematic processing may individuate counterstereotypical individuals, it also promotes an inconsistency resolution process that affects their evaluation.

More generally, the present research provides a theoretical and empirical bridge between research that demonstrates a negative bias against counterstereotypical individuals (e.g., Rudman, 1998; Schimel et al., 1999) and research that demonstrates a positive bias in favor of unique stimuli (Kim \& Markus, 1999). Westerners like unique stimuli, counterstereotypical individuals represent relatively unique stimuli, and adjectivallydescribed counterstereotypical individuals appear to be processed in a way that allows them to be appreciated for their uniqueness rather than denigrated for their deviance.

Finally, our research has implications for reducing prejudice against counterstereotypical individuals. To illustrate, it is known that prejudice against counterstereotypical job applications has the potential to influence hiring decisions (Gill, 2004). Hence, guidelines could be developed that advise counterstereotypical job applicants about the best way to describe themselves in their job applications in order to avoid discrimination from their prospective employer. These guidelines could provide advice to women who are applying for male-dominated jobs and men who are applying for femaledominated jobs. In both cases, the advice would be for applicants to describe themselves using more traits than behaviors in order to reduce any potential prejudice based on their counterstereotypical status.

\section{Limitations}

One limitation of the present research is that we did not investigate processing depth as a potential moderator of our effects. We predict that enhancing systematic processing will result in a reversal of the linguistic description effect due to the introduction of attribution processes. To explain, if people are encouraged to think carefully and systematically as they read person descriptions, then both behavior and trait descriptions should result in negative evaluations of counterstereotypical individuals because systematic processing promotes a stereotype inconsistency process that highlights the negative aspects of stereotype disconfirmation. However, behavior descriptions should result in less negative evaluations than trait descriptions because they provide a greater opportunity to explain away the stereotype disconfirmation. In particular, behavior descriptions (e.g., "a man who is crying") 
allow people to create subjective explanations for stereotype disconfirmation that dismiss it as a one-off, situation-specific exception to the rule (e.g., the man is crying for the first and last time in his life because he is grieving over his dead daughter). In contrast, trait descriptions (e.g., "a sensitive man") are more difficult to dismiss because they imply that the stereotype disconfirmation is relatively stable across time and situations (Douglas \& Sutton, 2003). Consequently, when people think carefully and systematically, they should evaluate counterstereotypical individuals negatively, but they should evaluate behavior descriptions of counterstereotypical individuals less negatively than trait descriptions. It is important to test this prediction of a reversed effect of linguistic description in order to identify the limits of our approach for reducing prejudice against counterstereotypical people.

A second limitation of the present research is that it did not include a manipulation of the valence of the descriptions that we used. Future research should manipulate, rather than counterbalance, this factor in order to investigate whether the moderating effect of linguistic bias generalizes across positive and negative descriptions. In addition, given that people process negative information more carefully than positive information (Baumeister, Bratslavsky Finkenauer, \& Vohs, 2001; ter Doest et al., 2002), it is possible that valence interacts with linguistic description to predict evaluations of counterstereotypical individuals. In particular, it is possible that negative behavioural descriptions are most likely to trigger an inconsistency resolution process. Again, future research should investigate this possibility. 


\section{References}

Baron, R. M., \& Kenny, D. A. (1986). The moderator-mediator variable distinction in social psychological research: Conceptual, strategic, and statistical considerations. Journal of Personality and Social Psychology, 51, 1173-1182.

Baumeister, R. F., Bratslavsky, E., Finkenauer, C., \& Vohs, K. D. (2001). Bad is stronger than good. Review of General Psychology 5, 323-370.

Bettencourt, B. A., Dill, K. E., Greathouse, S. A., Charlton, K., \& Mulholland, A. (1997). Evaluations of ingroup and outgroup members: The role of category-based expectancy violation. Journal of Experimental Social Psychology, 33, 244-275.

Biernat, M., Collins, E. C., Katzarska-Miller, I., \& Thompson, E. R. (2009). Race-based shifting standards and racial discrimination. Personality and Social Psychology Bulletin, 35, 16-28.

Biernat, M., Manis, M., \& Nelson, T. E. (1991). Stereotypes and standards of judgment. Journal of Personality and Social Psychology, 60, 485-499.

Brewer, M. B. (1988). A dual process model of impression formation. In R. S. Wyer \& T. K. Srull (Eds.), Advances in social cognition (Vol. 1, pp. 1-44). Mahwah, NJ: Erlbaum.

Brewer, M. B., \& Feinstein, A. (1999). Dual processes in the representation of persons and social categories. In S. Chaiken \& Y. Trope (Eds.), Dual process theories in social psychology (pp. 255-270). New York: Guilford Press.

Brewer, M. B. (1991). The social self: On being the same and different at the same time. Personality and Social Psychology Bulletin 17, 475482.

Cohen, T. R., Hall, D. L., \& Tuttle, J. (2009). Attitudes toward stereotypical versus counterstereotypical gay men and lesbians. Journal of Sex Research, 46, 274-281.

Coull, A., Yzerbyt, V. Y., Castano, E., Paladino, M. -P., Leemans, V. (2001). Protecting the ingroup: Motivated allocation of cognitive resources in the presence of threatening ingroup members. Group Processes \& Intergroup Relations, 4, 327-339.

Crandall, C. S., \& Eshleman, A. (2003). A justification-suppression model of the expression and experience of prejudice. Psychological Bulletin, 129, 414-446.

Douglas, K. M., \& Sutton, R. M. (2003). Effects of communication goals and expectancies on language abstraction. Journal of Personality and Social Psychology, 84, 682-696.

Eagly, A. H., \& Wood, W. (1982). Inferred sex differences in status as a determinant of gender stereotypes about social influence. Journal of Personality and Social Psychology, 43, 915-928.

Garcia-Marques, L., \& Mackie, D. M. (1999). The impact of stereotype-incongruent information on perceived group variability and stereotype change. Journal of Personality and Social Psychology, 77, 979-990.

Gutierrez, A. S., \& Unzueta, M. M. (2010). The effect of interethnic ideologies on the likability of stereotypic vs. counterstereotypic minority targets. Journal of Experimental Social Psychology, 46, 775-784.

Hewstone, M., Rubin, M., \& Willis, H. (2002). Intergroup bias. Annual Review of Psychology, 53, 575-604.

Hilton, J. L., \& von Hippel, W. (1996). Stereotypes. Annual Review of Psychology, 47, 237-271.

Holmes, J. D. (2009). Transparency of self-report racial attitude scales. Basic and Applied Social Psychology, 31, 95-101.

Hutter, R. R., \& Crisp, R. J. (2005). The composition of category conjunctions. Personality and Social Psychology Bulletin, 31, 647-657.

Hutter, R. R., \& Crisp, R. J. (2006). Implications of cognitive busyness for the perception of category conjunctions. Journal of Social Psychology, 146, 253-256. 
Imhoff, R., \& Erb, H. -P. (2009). What motivates nonconformity? Uniqueness seeking blocks majority influence. Personality and Social Psychology Bulletin, 35, 309-320.

Judd, C. M., Kenny, D. A., \& McClelland, G. H. (2001). Estimating and testing mediation and moderation in within-subject designs. Psychological Methods, 6, 115-134.

Kim, H., \& Markus, H. R. (1999). Deviance or uniqueness, harmony or conformity? A cultural analysis. Journal of Personality and Social Psychology, 77, 785-800.

Kunda, Z., Miller, D. T., \& Claire, T. (1990). Combining social concepts: The role of causal reasoning. Cognitive Science, 14, 551-577.

Levy, S. R., Stroessner, S. J., \& Dweck, C. S. (1998). Stereotype formation and endorsement: The role of implicit theories. Journal of Personality and Social Psychology, 74, 14211436.

Lynn, M. \& Snyder, C. R. (2002). Uniqueness seeking. In C. R. Snyder \& S. Lopez (Eds.), Handbook of Positive Psychology (pp. 395-410). Oxford: Oxford University Press.

Maass, A., Cadinu, M., Boni, M., \& Borini, C. (2005). Converting verbs into adjectives: Asymmetrical memory distortions for stereotypic and counterstereotypic information. Group Processes and Intergroup Relations, 8, 271-290.

Moreno, K. N., \& Bodenhausen, G. V. (1999). Resisting stereotype change: The role of motivation and attentional capacity in defending social beliefs. Group Processes \& Intergroup Relations, 2, 5-16.

Mullen, B., Brown, R., \& Smith, C. (1992). Ingroup bias as a function of salience, relevance and status: An integration. European Journal of Social Psychology, 22, 103-122.

Muller, D., Judd, C. M., \& Yzerbyt, V. Y. (2005). When moderation is mediated and mediation is moderated. Journal of Personality and Social Psychology, 89, 852-863.

Neuberg, S. L., \& Newsom, J. T. (1993). Personal need for structure: Individual differences in the desire for simple structure. Journal of Personality and Social Psychology, 65, 113-131.

Okimoto, T. G., \& Brescoll, V. L. (2010). The price of power: Power seeking and backlash against female politicians. Personality and Social Psychology Bulletin, 36, 923-936.

Pronin, E., Berger, J., \& Molouki, S. (2007). Alone in a crowd of sheep: Asymmetric perceptions of conformity and their roots in an introspection illusion. Journal of Personality and Social Psychology, 92, 585-595.

Rubin, M., Hewstone, M., \& Voci, A. (2001). Stretching the boundaries: Strategic perceptions of intragroup variability. European Journal of Social Psychology, 31, 413-429.

Rubin, M., Paolini, S., \& Crisp, R. J. (2010). A processing fluency explanation of bias against migrants. Journal of Experimental Social Psychology, 46, 21-28.

Rudman, L. A. (1998). Self-promotion as a risk factor for women: The costs and benefits of counterstereotypical impression management. Journal of Personality and Social Psychology, 74, 629-645.

Rudman, L. A., \& Fairchild, K. (2004). Reactions to counterstereotypic behavior: The role of backlash in cultural stereotype maintenance. Journal of Personality and Social Psychology, 87, 157176.

Rudman, L. A., \& Glick, P. (1999). Feminized management and backlash toward agentic women: The hidden costs to women of a kinder, gentler image of middle managers. Journal of Personality and Social Psychology, 77, 1004-1010.

Rudman, L. A., \& Glick, P. (2001). Prescriptive gender stereotypes and backlash toward agentic women. Journal of Social Issues, 57, 743-762.

Schimel, J., Simon, L., Greenberg, J., Pyszczynski, T., Solomon, S., Waxmonsky, J., \& Arndt, J. (1999). Stereotypes and terror management: Evidence that mortality salience 
enhances stereotypic thinking and preferences. Journal of Personality and Social Psychology, 77, 905-926.

Simon, B., Glässner-Bayerl, B., \& Stratenwerth, I. (1991). Stereotyping and self-stereotyping in a natural intergroup context: The case of heterosexual men and homosexual men. Social Psychology Quarterly, 54, 252-266.

Snyder, C. R., \& Fromkin, H. L. (1977). Abnormality as a positive characteristic: The development and validation of a scale measuring need for uniqueness. Journal of Abnormal Psychology, 86, 518-527.

Snyder, C. R., \& Fromkin, H. L. (1980). Uniqueness: The human pursuit of difference. New York: Plenum Press.

Ter Doest, L., Semin, G. R., \& Sherman, S. J. (2002). Linguistic context and social perception: Does stimulus abstraction moderate processing style? Journal of Language and Social Psychology, 21, 195-229.

Winkielman, P., Halberstadt, J., Fazendeiro, T., \& Catty, S. (2006). Prototypes are attractive because they are easy on the mind. Psychological Science, 17, 799-806.

Yzerbyt, V. Y., Muller, D., \& Judd, C. M. (2004). Adjusting researchers' approach to adjustment: On the use of covariates when testing interactions. Journal of Experimental Social Psychology, 40, 424-431. 


\section{Footnotes}

${ }^{1}$ At the beginning of the research questionnaire, participants completed the 11-item Personal Need for Structure scale (Thompson, Naccarato, \& Parker, 1989, cited in Neuberg $\&$ Newsom, 1993), four original items measuring the need for structure, and the three-item Person Theory Scale (Levy, Stroessner, \& Dweck, 1998). None of these measures provided any theoretically informative result. For the sake of brevity, we do not discuss these measures any further.

${ }^{2}$ Before they completed their ratings of target individuals, we explained to participants that conventional meant "how well the person matches society's conventions (social rules and practices)", stereotypical meant "how consistent or inconsistent (counterstereotypical) the person is with social stereotypes", and likable meant "how much you think that you would like or dislike the person if you met them in real life".

${ }^{3}$ It was not appropriate to compute indices of internal reliability for the likability ratings because these ratings represented single ratings of different targets (e.g., "a sensitive woman" and "an aggressive man") rather than multiple ratings of the same targets.

${ }^{4}$ As a further illustration of the effectiveness of this counterbalancing approach, consider people's preferences for fruit of different colors. If people prefer yellow tomatoes over red tomatoes, then this preference may be because either (1) people prefer yellow fruit more than red fruit, or (2) people prefer fruit that has an atypical color. However, if people's average evaluation of yellow tomatoes and red bananas is higher than their average evaluation of red tomatoes and yellow bananas, then this preference cannot be explained as a preference for yellow fruit because yellow fruit is equally represented in each category (i.e., yellow tomatoes in the atypical category and yellow bananas in the typical category). Instead, this preference can be explained as a preference for fruit that has an atypical color. In the present research, we used the same counterbalancing principle in order to analyze people's evaluations of stereotypical and counterstereotypical individuals.

${ }^{5}$ Our measure of familiarity showed large, positive correlations with our measures of stereotypicality, $r(237)=.75, p<.001$, and conventionality, $r(237)=.75, p<.001$. These relations make sense because people are liable to be more familiar with conventional and stereotypical individuals than with unconventional and counterstereotypical individuals. Given these relations, we considered whether familiarity could be used as an additional measure of stereotypicality. Consistent with this possibility, familiarity, stereotypicality, and conventionality combined together to form reliable indices for all eight types of target individuals ( $\alpha$ s ranged from .66 to .88). Using these indices, we found that stereotypical individuals were rated as being significantly more typical than counterstereotypical individuals for all four types of target individuals, $p \mathrm{~s}<.001$. This evidence provides further confirmation of the validity of the adjectives and behaviors that we used.

${ }^{6}$ Participants' gender did not qualify the key results in either this analysis or the subsequent analysis of processing fluency ( $p s \geq .186)$. Consequently, we reported analyses that excluded this factor.

${ }^{7}$ To test the influence of demand characteristics in our research, we included Rubin et al.'s (2010) Perceived Awareness of the Research Hypotheses scale at the end of our questionnaire. We included the average of the four PARH items $(\alpha=.82)$ as a covariate in our analyses investigating the evaluation of target individuals. All of the key effects remained significant $(p \mathrm{~s} \leq .010)$, and the PARH index did not act as a significant covariate ( $p s \geq .17$ ). Hence, participants' perceived awareness of the research hypothesis did not account for the significant effects that we had obtained.

${ }^{8}$ Judd, Kenny, and McClelland (2001, p. 131) recommended summing the different levels of repeated measures variables and then centering the sum score in order to test the moderating effect of such variables in mediation analyses. In the present research, we 
summed processing fluency scores for adjectival and behavioral descriptions in order to create separate centered sum scores for gender targets $(\alpha=.65)$ and sexuality targets $(\alpha=$ $.65)$. 